News:

\title{
Macrophage-produced IL-10 limits the chemotherapy efficacy in breast cancer
}

\author{
Xinguo JIANG \\ (Department of Medicine, VA Palo Alto Health Care System/Stanford \\ University School of Medicine, Stanford, CA 94305, USA) \\ E-mail: xinguoj@stanford.edu \\ doi:10.1631/jzus.B1400352
}

Macrophages are among the most abundant immune cells in the tumor microenvironment, where they are known as tumor-associated macrophages (TAMs). Substantial evidence indicates that TAMs generally play protumoral roles in the primary as well as metastatic sites (Noy and Pollard, 2014). TAMs are also known to interfere with several tumor therapeutic modalities, such as chemotherapy, irradiation, and immunotherapy. In general, depletion of M2-like TAMs or reprogramming them into M1-like phenotype enhances the efficacy of these therapies (Rolny et al., 2011; de Palma and Lewis, 2013; Germano et al., 2013).

Chemotherapy has been shown to increase macrophage infiltration in human breast cancer tissues and the ratio of CD68/CD8 expression predicts the patient response to the treatment (DeNardo et al., 2011). TAMs have been known to promote breast cancer progression and increase therapy resistance through mechanisms such as the enhancement of angiogenesis, tumor cell proliferation and invasiveness by producing growth factors such as vascular endothelial growth factor (VEGF), epidermal growth factor (EGF) and FGF2 (Jiang, 2014). However, improved efficacy of chemotherapy by the means of TAM depletion has been shown to be dependent on the enhanced anti-tumor activity of $\mathrm{CD} 8^{+} \mathrm{T}$ cells (DeNardo et al., 2011). How TAM suppresses CD ${ }^{+}$

(D) ORCID: Xinguo JIANG, http://orcid.org/0000-0003-3266-9238 (C) Zhejiang University and Springer-Verlag Berlin Heidelberg 2015
$\mathrm{T}$ cells was poorly known. Using two mouse breast cancer models, Ruffell et al. (2014) recently demonstrated that chemotherapy-induced macrophage infiltration suppresses dendritic cell (DC) production of IL-12, which in turn inhibits $\mathrm{CD} 8^{+} \mathrm{T}$ cell activity, and limits the efficacy of the chemotherapy. They first showed that macrophages express the highest levels of IL-10 among tumor infiltrating leukocytes, including Treg cells, which are known to be a major source of IL-10 in other murine tumor models (Stewart et al., 2013). IL-10 signaling blockade with an anti-IL-10R mAb improves the efficacy of paclitaxel and carboplatin therapies in the mouse mammary tumor virus-polyoma middle T (MMTV-PyMT) luminal B-type and the C3(1)-TAg triple negative breast cancer models, suggesting that IL-10 limiting the chemotherapy efficacy is a generalizable phenomenon in breast cancers. Anti-IL-10R mAb treatment was next shown to increase the number of infiltrated $\mathrm{CD}^{+} \mathrm{T}$ cells in the tumor microenvironment, and depletion of $\mathrm{CD} 8^{+} \mathrm{T}$ cells ablated the beneficial effect of anti-IL-10R mAb, confirming that the therapeutic efficacy of anti-IL-10R mAb depends on the activity of $\mathrm{CD}^{+} \mathrm{T}$ cells. Ruffell et al. (2014) went on to show that anti-IL-10R mAb treatment does not affect macrophage recruitment as well as its polarization and function following chemotherapy; in addition, anti-IL-10R mAb also does not directly alter the proliferation and function of $\mathrm{CD} 8^{+} \mathrm{T}$ cells, suggesting that IL-10 may indirectly suppress $\mathrm{CD} 8^{+} \mathrm{T}$ cell activity by acting on another cell type. Indeed, they found that tumor infiltrating DCs express much high levels of IL-10R than that of $\mathrm{CD} 8^{+} \mathrm{T}$ cells. They next demonstrated that IL-10 suppresses DC production of IL-12, and blockade of the IL-12/IL-12R pathway abolished the beneficial effect of anti-IL-10R $\mathrm{mAb}$. Because IL-12 is a cytokine known to promote $\mathrm{CD} 8^{+} \mathrm{T}$ cell proliferation and its effector function 
(Trinchieri, 2003), a novel mechanism by which macrophage suppresses the chemotherapeutic efficacy was established. This mechanism is that macrophageproduced IL-10 suppresses DC expression of IL-12, which then leads to decreased $\mathrm{CD} 8^{+} \mathrm{T}$ cell infiltration and activity and increased chemotherapy resistance. Ruffell et al. (2014) further validated some of their preclinical findings in patients. They showed that increased IL-10 expression is common in human breast cancer tissues, and high expression levels of IL-12A are associated with a better pathological complete response to chemotherapy.

In summary, Ruffell et al. (2014) have provided compelling evidence showing that the IL-10/IL-10R and IL-12/IL-12R pathways may play essential roles in the pathological response following breast cancer chemotherapy-induced TAM recruitment, and targeting these pathways may decrease chemotherapy resistance and improve the overall outcome.

\section{References}

de Palma, M., Lewis, C.E., 2013. Macrophage regulation of tumor responses to anticancer therapies. Cancer Cell, 23(3):277-286. [doi:10.1016/j.ccr.2013.02.013]

DeNardo, D.G., Brennan, D.J., Rexhepaj, E., et al., 2011. Leukocyte complexity predicts breast cancer survival and functionally regulates response to chemotherapy. Cancer Discov., 1(1):54-67. [doi:10.1158/2159-8274.CD-10-0028]

Germano, G., Frapolli, R., Belgiovine, C., et al., 2013. Role of macrophage targeting in the antitumor activity of trabectedin. Cancer Cell, 23(2):249-262. [doi:10.1016/j. ccr.2013.01.008]

Jiang, X., 2014. Harnessing the immune system for the treatment of breast cancer. J. Zhejiang Univ.-Sci. B (Biomed. \& Biotechnol.), 15(1):1-15. [doi:10.1631/jzus.B1300264]
Noy, R., Pollard, J.W., 2014. Tumor-associated macrophages: from mechanisms to therapy. Immunity, 41(1):49-61. [doi:10.1016/j.immuni.2014.06.010]

Rolny, C., Mazzone, M., Tugues, S., et al., 2011. HRG inhibits tumor growth and metastasis by inducing macrophage polarization and vessel normalization through downregulation of PlGF. Cancer Cell, 19(1):31-44. [doi:10.1016/ j.ccr.2010.11.009]

Ruffell, B., Chang-Strachan, D., Chan, V., et al., 2014. Macrophage IL-10 blocks $\mathrm{CD}^{+} \mathrm{T}$ cell-dependent responses to chemotherapy by suppressing IL-12 expression in intratumoral dendritic cells. Cancer Cell, 26(5):623-637. [doi:10.1016/j.ccell.2014.09.006]

Stewart, C.A., Metheny, H., Iida, N., et al., 2013. Interferondependent IL-10 production by Tregs limits tumor Th17 inflammation. J. Clin. Invest., 123(11):4859-4874. [doi:10. 1172/JCI65180]

Trinchieri, G., 2003. Interleukin-12 and the regulation of innate resistance and adaptive immunity. Nat. Rev. Immunol., 3(2):133-146. [doi:10.1038/nri1001]

\section{中文概要}

题 目: 巨噬细胞产生的白介素-10 降低乳腺癌的化疗疗效

概 要: 本文简单介绍了最近发表在《肿瘤细胞》(Cancer Cell) 上的一篇关于如何提高乳腺癌化疗疗效的 文章。这篇文章发现了乳腺癌化疗可以导致促肿 瘤生长的巨唻细胞数量的增多, 这些巨噬细胞可 以产生大量的白介素-10（IL-10），从而抑制肿 瘤内树突状细胞 IL-12 的产生, 由此抑制具细胞 毒性的 $\mathrm{CD} 8^{+} \mathrm{T}$ 细胞的功能, 最终降低化疗疗效。 除此之外, 文章还证明了阻断 IL-10 的功能可以 促进乳腺癌化疗效果。这篇文章因此提出了可以 降低乳腺癌化疗耐受的一种新的治疗靶点。

关键词: 乳腺癌; 化疗; 巨喍细胞; IL-10; IL-12; 树突 状细胞 